\title{
Instrument-independent specification of the diffraction geometry and polarization state of the incident $X$-ray beam
}

\author{
Marc Schiltz and Gérard Bricogne
}

J. Appl. Cryst. (2009). 42, 101-108

Copyright (C) International Union of Crystallography

Author(s) of this paper may load this reprint on their own web site or institutional repository provided that this cover page is retained. Republication of this article or its storage in electronic databases other than as specified above is not permitted without prior permission in writing from the IUCr.

For further information see http://journals.iucr.org/services/authorrights.html

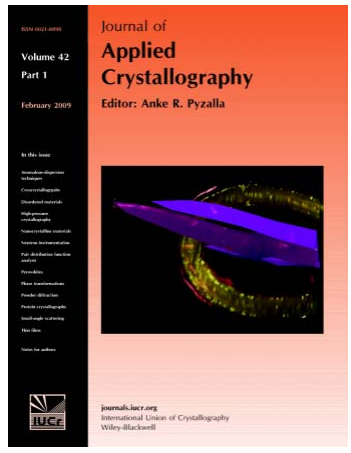

Many research topics in condensed matter research, materials science and the life sciences make use of crystallographic methods to study crystalline and non-crystalline matter with neutrons, X-rays and electrons. Articles published in the Journal of Applied Crystallography focus on these methods and their use in identifying structural and diffusioncontrolled phase transformations, structure-property relationships, structural changes of defects, interfaces and surfaces, etc. Developments of instrumentation and crystallographic apparatus, theory and interpretation, numerical analysis and other related subjects are also covered. The journal is the primary place where crystallographic computer program information is published.

Crystallography Journals Online is available from journals.iucr.org 
Journal of

Applied

Crystallography

ISSN 0021-8898

Received 26 September 2008

Accepted 18 November 2008

(C) 2009 International Union of Crystallography Printed in Singapore - all rights reserved

\section{Instrument-independent specification of the diffraction geometry and polarization state of the incident $X$-ray beam}

\author{
Marc Schiltz $^{a *}$ and Gérard Bricogne ${ }^{b}$ \\ aÉcole Polytechnique Fédérale de Lausanne (EPFL), Laboratoire de Cristallographie, CH-1015 \\ Lausanne, Switzerland, and ${ }^{\mathbf{b}}$ Global Phasing Ltd, Sheraton House, Castle Park, Cambridge CB3 \\ OAX, UK. Correspondence e-mail: marc.schiltz@epfl.ch
}

This work augments the proposal of Schwarzenbach \& Flack [J. Appl. Cryst. (1989), 22, 601-605], who have advocated the use of a diffractometerindependent definition of the azimuthal angle $\psi$ to specify the diffraction geometry of a Bragg reflection. It is here proposed that one additional angle $\xi$, which is also based on a diffractometer-independent definition, is needed to encode the direction of linear polarization for those experiments where this quantity is of importance. This definition is then extended to the cases of partially and/or elliptically polarized X-ray beams, and the use of three normalized Stokes parameters, $P_{1}, P_{2}$ and $P_{3}$, together with $\xi$, is advocated in order to characterize exhaustively the polarization state of the incident beam. The conventions proposed here present a general, unambiguous and economical means of encoding the information about the diffraction geometry, without the need to record any further information about the instrument, crystal orientation matrix and goniometer angles. Data-processing software using these definitions to analyse polarization-dependent phenomena becomes instrument-independent and completely general. These methods have been implemented in the macromolecular phasing program $S H A R P$ for exploiting the polarization anisotropy of anomalous scattering in protein crystals.

\section{Introduction}

The polarization properties of the X-ray beam are of importance in a number of diffraction experiments, including magnetic diffraction (Lovesey \& Collins, 1996) and polarized resonant (anomalous) diffraction (Templeton \& Templeton, 1982; Dmitrienko et al., 2005). We have recently shown that the polarization anisotropy of anomalous scattering (AAS) is a significant and ubiquitous effect in protein crystallography and that its exploitation can substantially enhance the phasing power of single- or multi-wavelength anomalous diffraction measurements collected at or near an absorption edge (Schiltz \& Bricogne, 2008). In these experiments, the polarization properties of the incident X-ray beam enter as parameters into the structure factor equations. We have for instance shown that, in this context, the anomalous scattering factor of an atom that displays AAS can in many cases be approximated by

$$
f=\left({ }^{\mathrm{t}} \mathbf{p}^{\prime} \mathbf{F} \mathbf{p}\right) /\left({ }^{\mathrm{t}} \mathbf{p}^{\prime} \mathbf{p}\right),
$$

where the left superscript $t$ stands for matrix transposition, $\mathbf{F}$ is a second-rank tensor and it is assumed that the incident beam is completely linearly polarized along a direction given by the unit vector $\mathbf{p}$. The direction of unit vector $\mathbf{p}^{\prime}$ is that of the projection of $\mathbf{p}$ onto a plane perpendicular to the scattered beam direction and corresponds to the direction of linear polarization of the diffracted beam in the absence of AAS. To refine the tensorial values of the anomalous scattering factors and/or to extract phase information from AAS-induced symmetry-breaking effects (Schiltz \& Bricogne, 2008), it is therefore necessary to record information about the orientation of the crystal with respect to the direction of X-ray polarization for each reflection measurement.

The experimental geometry of a Bragg reflection in X-ray diffraction is not completely specified by the lattice constants of the crystal and the reciprocal lattice indices $(h k l)$ of the scattering vector. Almost 20 years ago, Schwarzenbach \& Flack (1989) advocated a diffractometer-independent definition of the azimuthal angle $\psi$ to specify, for each experimental reflection measurement, the orientation of the incident and diffracted X-ray beams with respect to the crystal lattice. They noted that the values of diffractometer-based $\psi$ angles become meaningless without the additional specification of the crystal orientation matrix and the definition of the goniometer setting angles and senses of rotation of the various circles. On the other hand, with a crystal-based definition of $\psi$, the diffraction geometry can be specified by four values, namely $h, k, l$ and $\psi$, without the need for any additional information about the instrument or crystal orientation matrix. The specification of an azimuthal angle $\psi$ allows one to carry out the calculation and/or refinement of absorption, thermal diffuse scattering 
and anisotropic extinction corrections. A further area of applications is that of multiple-beam X-ray diffraction experiments, where $\psi$-scan profiles can be used in the determination of absolute structures (Hümmer \& Weckert, 1995) or for the computation of triplet phases (Weckert \& Hümmer, 1997). However, for experiments that directly involve the polarization of the incident X-ray beam, the mere indication of the azimuthal angle $\psi$ is not enough to specify the diffraction geometry completely, even in the simple case of linear polarization.

For conventional diffraction data collection, area detectors are now universally used in macromolecular crystallography and also to a very large extent in small-molecule crystallography. In these experiments, a large number of reflections are recorded nearly simultaneously. No $\psi$-scan is performed and the geometry is such that the direction of polarization of the X-ray beam will be in a general orientation (neither parallel nor perpendicular) with respect to the diffraction plane. It therefore becomes necessary to compute and encode the direction of polarization for each of these reflection measurements. With the current emphasis on high-throughput data collection schemes at synchrotrons, where large quantities of data can be collected in a short time, it becomes imperative to keep track of all geometric information about the experiment and to encode it in an efficient and easily usable way. In the present communication we propose that one additional angle $\xi$, which is based on a diffractometerindependent definition, can fulfil the task of recording the direction of polarization. We then extend this discussion to the cases of partially and/or elliptically polarized X-ray beams and advocate the use of three normalized Stokes parameters, $P_{1}$, $P_{2}$ and $P_{3}$, together with $\xi$, to characterize exhaustively the most general state of polarization of the incident beam.

\section{Diffractometer-independent specification of diffraction geometry}

The vectors and tensors involved in the structure factor equations reported by Schiltz \& Bricogne (2008) [equation (1)] are expressed in a crystal Cartesian basis. On the other hand, the direction of the X-ray beam polarization is usually known in some laboratory reference system, where it is often fixed. It would therefore seem necessary to record the information about the orientation of the crystal at each diffraction measurement with respect to this laboratory system. As an alternative, following Schwarzenbach \& Flack (1989), our guiding principle has been that all geometric quantities should be defined with respect to the crystal lattice, not the laboratory.

\subsection{Definition of the $\xi$ angle}

Fig. 1 shows the relevant vectors and angles defined by Schwarzenbach \& Flack (1989) and (in colour) the new additional geometric objects that are needed for our definition. All vectors shown are unit vectors: $\mathbf{s}$ and $\mathbf{s}^{\prime}$ represent the incident and diffracted beam directions, respectively [these are, respectively, designated $\mathbf{p}$ and $\mathbf{d}$ by Schwarzenbach \& Flack (1989), but we prefer here to avoid the use of $\mathbf{p}$ in order to prevent any possible confusion with the direction of beam polarization]; $\mathbf{e}$ is collinear with $-\left(\mathbf{s}+\mathbf{s}^{\prime}\right)$; $\mathbf{f}$ is collinear with the scattering vector $\mathbf{H}=h \mathbf{a}^{*}+k \mathbf{b}^{*}+l \mathbf{c}^{*} ; \mathbf{g}$ is perpendicular to the diffraction plane and chosen such that $(\mathbf{e}, \mathbf{f}, \mathbf{g})$ forms a right-handed coordinate system. The vector $\mathbf{q}=\mathbf{Q} /|\mathbf{Q}|$ indicates the reference direction defining the zero position of $\psi$ by the convention given by Schwarzenbach \& Flack (1989):

$$
\mathbf{Q}= \begin{cases}h \mathbf{a}-h \mathbf{b} & \text { if } h=k=l \\ (k-l) \mathbf{a}+(l-h) \mathbf{b}+(h-k) \mathbf{c} & \text { otherwise }\end{cases}
$$

We now define another reference vector $\mathbf{t}$ lying in the diffraction plane $(\mathbf{e}, \mathbf{f})$ and perpendicular to the incident beam direction $\mathbf{s}$. Furthermore, $\mathbf{t}$ is defined to point upwards; $\mathbf{t} \cdot \mathbf{f}$ is always positive. Thus

$$
\mathbf{t}=-\sin \theta \mathbf{e}+\cos \theta \mathbf{f} .
$$

It follows from this definition that the plane $(\mathbf{t}, \mathbf{g})$ is perpendicular to the incident beam direction $\mathbf{s}$. Thus, if the incident beam is linearly polarized along a direction given by the unit vector $\mathbf{p}$, this vector is necessarily contained in the $(\mathbf{t}, \mathbf{g})$ plane. $\xi$ then defines the angle between $\mathbf{p}$ and the reference vector $\mathbf{t}$ :

$$
\begin{aligned}
\cos \xi & =\mathbf{p} \cdot \mathbf{t}, \\
\sin \xi & =\mathbf{p} \cdot \mathbf{g} .
\end{aligned}
$$

This definition of $\xi$ uniquely specifies the vector $\mathbf{p}$ by

$$
\mathbf{p}=\cos \xi \mathbf{t}+\sin \xi \mathbf{g} .
$$

The vectors $\mathbf{s}$ and $\mathbf{p}$ are usually available as components in some laboratory-fixed coordinate system (where they are constant in most cases). The computational steps necessary to convert them to components in a crystal-based coordinate system using the goniometer angles recorded for each reflection are outlined in Appendix B. This requires knowledge of the definition of the laboratory-fixed coordinate system (some conventions used by area-detector processing programs are summarized in Appendix $B$ ), the crystal orientation matrix, the design of the goniometer instrument, and the conventions used to define the senses of rotation and zero positions of the

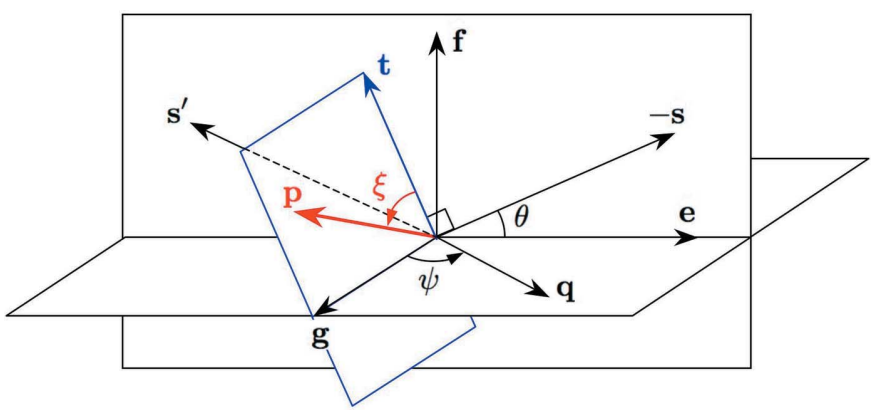

Figure 1

Diffraction geometry. The figure displays the various unit vectors and angles defined by Schwarzenbach \& Flack (1989). The new additional geometric objects that are needed for the definition presented in this paper are displayed in colour. 
goniometer circles. It would therefore be highly desirable if the $\psi$ and $\xi$ values were computed during the integration of the area-detector diffraction images when all the necessary information is readily available, and we strongly call for developers to include these computations in their software packages. The computation of the azimuthal angle $\psi$ using the Schwarzenbach \& Flack (1989) convention is already available as an option in the data-processing program $X D S$ (Kabsch, 1988), but to our knowledge, this facility has not been implemented in any of the other commonly used area-detector processing programs.

\subsection{Discussion}

Once the six values $h, k, l, \theta, \psi$ and $\xi$ have been recorded in the reflection file, the diffraction geometry can be uniquely derived for each reflection, without the need to specify any additional information about the instrument, goniometer angles or crystal orientation. The vectors $\mathbf{s}, \mathbf{s}^{\prime}$ and $\mathbf{p}$, which represent directions of physical quantities, can be computed by the formulae that are summarized in Appendix $C$.

An alternative proposal would be to specify the vectors $\mathbf{s}, \mathbf{s}^{\prime}$ and $\mathbf{p}$ through their direction cosines. A number of data processing programs already have an option to compute the direction cosines of $\mathbf{s}$ and $\mathbf{s}^{\prime}$ and write them to the reflection file. These values can then be used in the refinement of an empirical absorption surface as proposed by Blessing (1995). However, direction cosines necessarily refer to certain coordinate axes, which must then be specified to ensure that the same convention is used by the data-processing software that computes and writes the direction cosines to the reflection file and the software that uses these values. ${ }^{1}$ On the other hand, the definition of the $\theta, \psi$ and $\xi$ angles is completely independent of any coordinate system. The specification of the $\mathbf{s}, \mathbf{s}^{\prime}$ and $\mathbf{p}$ vectors through their direction cosines also requires the recording of more values (nine as opposed to three). We therefore assert that the specification of the three angles $\theta, \psi$ and $\xi$ is the most general, unambiguous and economical means to encode the information about the diffraction geometry.

In practice, it may even be superfluous to record the $\theta$ values explicitly as these can be computed, for a given reflection, from the $h k l$ indices and from the wavelength $\lambda$ of the incident X-ray beam. As an alternative to $\theta$, the value of $\lambda$ could be recorded. Since in most data sets the wavelength is constant for large batches of reflections, it is unnecessary to record the $\theta$ or $\lambda$ values individually for each reflection.

\subsection{Further extension}

As a further extension, we can also define a reference vector $\mathbf{t}^{\prime}$ lying in the diffraction plane (e, f) and perpendicular to the diffracted beam direction $\mathbf{s}^{\prime}$. We define $\mathbf{t}^{\prime}$ to point upwards; $\mathbf{t}^{\prime} \cdot \mathbf{f}$ is always positive. Thus

\footnotetext{
${ }^{1}$ As an example of possible confusion, the standard convention in the program SHELX (Sheldrick, 2008) is to use direction cosines defined relative to the crystal reciprocal lattice axes, whereas the software implementing the Blessing (1995) method uses direction cosines defined with respect to orthogonal crystal axes (alternative orthogonalization conventions can give rise to further confusion in such cases).
}

$$
\mathbf{t}^{\prime}=\sin \theta \mathbf{e}+\cos \theta \mathbf{f}
$$

If the incident X-ray beam is completely linearly polarized along $\mathbf{p}$ and in the absence of AAS, the scattered beam will also be completely linearly polarized along a direction denoted by the unit vector $\mathbf{p}^{\prime}$ and which is obtained by projecting $\mathbf{p}$ onto a plane perpendicular to the scattered beam direction $\mathbf{s}^{\prime}$. Thus,

$$
\mathbf{p}^{\prime}=\frac{\left(\mathbf{p} \cdot \mathbf{t}^{\prime}\right) \mathbf{t}^{\prime}+(\mathbf{p} \cdot \mathbf{g}) \mathbf{g}}{\left[\left(\mathbf{p} \cdot \mathbf{t}^{\prime}\right)^{2}+(\mathbf{p} \cdot \mathbf{g})^{2}\right]^{1 / 2}}=\frac{\cos \xi \cos (2 \theta) \mathbf{t}^{\prime}+\sin \xi \mathbf{g}}{\left[\cos ^{2} \xi \cos ^{2}(2 \theta)+\sin ^{2} \xi\right]^{1 / 2}} .
$$

It can be noted here that

$$
\left(\mathbf{p} \cdot \mathbf{p}^{\prime}\right)^{2}=\cos ^{2} \xi \cos ^{2}(2 \theta)+\sin ^{2} \xi
$$

is the conventional polarization correction factor for diffraction by a completely linearly polarized X-ray beam. The above definition for $\mathbf{p}^{\prime}$ is therefore not applicable when $2 \theta=90^{\circ}$ and $\xi=0^{\circ}$. This corresponds to scattering at $90^{\circ}$ in the plane of polarization, a geometry where the scattered intensity (in the absence of AAS) is zero.

Finally, we can define vectors $\mathbf{p}_{\perp}$ and $\mathbf{p}_{\perp}^{\prime}$ by

$$
\begin{aligned}
\mathbf{p}_{\perp} & =\mathbf{s} \times \mathbf{p}, \\
\mathbf{p}_{\perp}^{\prime} & =\mathbf{s}^{\prime} \times \mathbf{p}^{\prime},
\end{aligned}
$$

such that (s, p, $\left.\mathbf{p}_{\perp}\right)$ and $\left(\mathbf{s}^{\prime}, \mathbf{p}^{\prime}, \mathbf{p}_{\perp}^{\prime}\right)$ form right-handed orthogonal coordinate systems attached to the incident and diffracted beam directions, respectively. The computation of these additional vectors does not require anything more than knowledge of the cell parameters and, for each reflection, the $h k l$ indices and the three angles $\theta, \psi$ and $\xi$.

The scattering of X-rays from an atom that exhibits AAS is described by a matrix of four elements corresponding to polarization transfers from the incident beam polarization components along $\mathbf{u}$ and $\mathbf{v}$ to the scattered beam polarization components along the directions $\mathbf{u}^{\prime}$ and $\mathbf{v}^{\prime}$ (Templeton \& Templeton, 1982; Fanchon \& Hendrickson, 1990; Kirfel et al., 1991; Schiltz \& Bricogne, 2008):

$$
\begin{aligned}
& \Phi_{u^{\prime} u}={ }^{\mathrm{t}} \mathbf{u}^{\prime} \mathbf{F} \mathbf{u}, \\
& \Phi_{v^{\prime} u}={ }^{\mathrm{t}} \mathbf{v}^{\prime} \mathbf{F} \mathbf{u}, \\
& \Phi_{u^{\prime} v}={ }^{\mathrm{t}} \mathbf{u}^{\prime} \mathbf{F} \mathbf{v}, \\
& \Phi_{v^{\prime} v}={ }^{\mathrm{t}} \mathbf{v}^{\prime} \mathbf{F} \mathbf{v},
\end{aligned}
$$

where the unit vectors $\mathbf{u}$ and $\mathbf{v}$ are mutually perpendicular and perpendicular to the incident beam direction $\mathbf{s}$, while the unit vectors $\mathbf{u}^{\prime}$ and $\mathbf{v}^{\prime}$ are mutually perpendicular and perpendicular to the scattered beam direction $\mathbf{s}^{\prime}$. The choice of these directions is free, but we have demonstrated (Schiltz \& Bricogne, 2008) that the particular choice

$$
\mathbf{u}=\mathbf{p}, \quad \mathbf{v}=\mathbf{p}_{\perp}, \quad \mathbf{u}^{\prime}=\mathbf{p}^{\prime}, \quad \mathbf{v}^{\prime}=\mathbf{p}_{\perp}^{\prime}
$$

can considerably simplify the expressions for AAS in the case of linearly polarized X-rays. 


\section{Generalization to partially and/or elliptically polarized X-ray beams}

So far we have assumed that the incident X-ray beam is completely linearly polarized. On synchrotrons, the radiation emitted in the plane of the electron orbit is indeed linearly polarized, with the direction of polarization lying in that same plane. However, the radiation emitted from a bending magnet is elliptically polarized above and below the plane of the electron orbit (Bathow et al., 1966; Brunel et al., 1983; Materlik \& Suortti, 1984; Templeton \& Templeton, 1988). In most experiments, a fan of radiation of a certain angular range is intercepted and focused by optical devices onto the sample. Thus, the radiation that arrives on the crystal is not purely linearly polarized (although, with undulator insertion devices, the degree of linear polarization remains often well above 0.9 ). Reflecting optical elements such as crystal monochromators and focusing mirrors also modify the polarization state of the beam. With phase plates based on perfect crystals (e.g. diamond), it is also possible to actively tune the polarization state of the X-ray beam, to change the polarization plane from horizontal to vertical (or any other direction) or to convert linear polarization to circular polarization (Giles et al., 1994; Hirano et al., 1995). The radiation emitted from a laboratory $\mathrm{X}$-ray tube is completely unpolarized, but reflecting optics (monochromators, mirrors, capillary X-ray optics) will induce partial linear polarization. The mere indication of a direction $\mathbf{p}$ is therefore not sufficient to specify completely the polarization properties of an X-ray beam in the general case.

\subsection{Conventional ways of specifying the polarization properties of an X-ray beam}

The 'degree of polarization' is widely used as an additional quantity to specify the X-ray beam properties. Unfortunately this concept is not totally unequivocal. What is usually meant is the degree of linear polarization of the beam, defined in most cases by the ratio

$$
\mathcal{D}_{\text {lin }}=\left(I_{\mathrm{p}}-I_{\mathrm{n}}\right) /\left(I_{\mathrm{p}}+I_{\mathrm{n}}\right),
$$

where $I_{\mathrm{p}}$ is the intensity of the polarization component in the plane of polarization and $I_{\mathrm{n}}$ the intensity of the polarization component perpendicular to that plane. Note that this definition is only useful if a plane of polarization can be defined. In the case of elliptical polarization, the 'plane' of polarization can be defined to be the plane containing the major semi-axis of the ellipse. In the special case of circular polarization, this plane would be degenerate, but $\mathcal{D}_{\text {lin }}$ is anyway zero. However, for a completely unpolarized beam, $\mathcal{D}_{\text {lin }}$ is also zero, but the beam properties of an unpolarized beam are not the same as those of circularly polarized X-rays, and, in the presence of AAS, the scattered intensities differ in the two cases. The conclusion is that a single degree of (linear) polarization is not sufficient to specify completely the polarization properties of an X-ray beam that are needed in the case of AAS.

\subsection{Stokes parameters}

The Stokes (1852) parameters provide a means of specifying the most general polarization properties of a nearly monochromatic light beam (Born \& Wolf, 1959). They are now widely used in the field of magnetic X-ray scattering (Blume \& Gibbs, 1988; Lovesey \& Collins, 1996, 2001) but they have only been very rarely used in the context of conventional X-ray diffraction (Vaillant, 1977; Fanchon \& Hendrickson, 1990). They consist of a set of four real quantities, $S_{0}, S_{1}, S_{2}$ and $S_{3}$, that have the dimensions of intensity. The parameter $S_{0}$ corresponds to the total intensity of the X-ray beam. The parameter $S_{1}$ corresponds to the intensity difference between the linear polarization components along directions located, respectively, at 0 and $90^{\circ}$ with respect to some reference direction. The parameter $S_{2}$ corresponds to the intensity difference between the linear polarization components along directions located at 45 and $-45^{\circ}$ with respect to the reference direction. Finally, the parameter $S_{3}$ corresponds to the intensity difference between the right- and left-circular polarization components. $^{2}$ If the reference direction is denoted by $\mathbf{p}$ (perpendicular to $\mathbf{s}$ ), we can write

$$
\begin{aligned}
& S_{0}=I(\mathbf{p})+I\left(\mathbf{p}_{\perp}\right), \\
& S_{1}=I(\mathbf{p})-I\left(\mathbf{p}_{\perp}\right)
\end{aligned}
$$

and

$$
S_{2}=I\left(\mathbf{p}+\mathbf{p}_{\perp}\right)-I\left(\mathbf{p}-\mathbf{p}_{\perp}\right),
$$

where $I(\mathbf{u})$ denotes the intensity of the linear polarization component along the direction $\mathbf{u}$. A fundamental property of the Stokes parameters is given by the following inequality (Born \& Wolf, 1959):

$$
S_{0}^{2} \geq S_{1}^{2}+S_{2}^{2}+S_{3}^{2}
$$

where the equality holds for a completely polarized (not necessarily linearly polarized) beam.

Since we are only interested in the polarization properties of the X-ray beam, we can use the three normalized Stokes parameters, which are also sometimes called Stokes-Poincaré parameters since they correspond to the Cartesian coordinates of a point on the Poincaré (1889) sphere,

$$
P_{1}=S_{1} / S_{0}, \quad P_{2}=S_{2} / S_{0}, \quad P_{3}=S_{3} / S_{0} .
$$

As a consequence of equation (18), the values of $P_{1}, P_{2}$ and $P_{3}$ always fall between -1 and +1 .

The degree of polarization is given by

$$
\mathcal{D}=\left(S_{1}^{2}+S_{2}^{2}+S_{3}^{2}\right)^{1 / 2} / S_{0}=\left(P_{1}^{2}+P_{2}^{2}+P_{3}^{2}\right)^{1 / 2},
$$

the degree of linear polarization is given by

$$
\mathcal{D}_{\text {lin }}=\left(S_{1}^{2}+S_{2}^{2}\right)^{1 / 2} / S_{0}=\left(P_{1}^{2}+P_{2}^{2}\right)^{1 / 2}
$$

and the degree of circular polarization is given by

\footnotetext{
${ }^{2}$ The Stokes parameters can also be defined in terms of electromagnetic theory (Born \& Wolf, 1959) as $S_{0}=\left\langle E_{x}^{2}\right\rangle+\left\langle E_{y}^{2}\right\rangle, S_{1}=\left\langle E_{x}^{2}\right\rangle-\left\langle E_{y}^{2}\right\rangle, S_{2}=$ $2\left\langle E_{x} E_{y} \cos \delta\right\rangle$ and $S_{3}=2\left\langle E_{x} E_{y} \sin \delta\right\rangle$, where $E_{x}$ and $E_{y} \exp (i \delta)$ are projections of the electric field vector along two orthogonal directions $x$ and $y, \delta$ is the phase difference between them, and the angular brackets indicate timeaveraged quantities.
} 


$$
\mathcal{D}_{\text {circ }}=\left(S_{3}^{2}\right)^{1 / 2} / S_{0}=\left|P_{3}\right| .
$$

Thus, an unpolarized beam has normalized Stokes parameters $P_{1}=P_{2}=P_{3}=0$, whereas for a circularly polarized beam we have $P_{1}=P_{2}=0, P_{3}= \pm 1$, with the sign depending on the polarization sense $(+1$ for right-circular and -1 for leftcircular polarization).

In order to exploit the polarization properties of synchrotron radiation, in particular for experiments that aim at using AAS effects in macromolecular crystallography, it is necessary to characterize fully the polarization properties of the X-rays delivered at a given beamline. Several methods have been described in the literature to determine experimentally the three normalized Stokes parameters of a synchrotron beam in the hard X-ray region (Ishikawa et al., 1991; Shen \& Finkelstein, 1992; Hirano et al., 1995). We call for beamline scientists to carry out such measurements and report the polarization properties of their beamlines in terms of Stokes parameters.

Even though Stokes parameters are a general means to specify the polarization properties of an X-ray beam, it is clear that, for a given polarization state, the values of the normalized Stokes parameters $P_{1}$ and $P_{2}$ (but not of the parameter $P_{3}$ ) depend on the particular choice of the reference direction p. We propose that $\mathbf{p}$ be an arbitrarily chosen direction perpendicular to $\mathbf{s}$ and fixed in the laboratory frame of reference. Once the reference vector $\mathbf{p}$ has been chosen, the Stokes parameters will be identical for all reflections recorded under similar X-ray beam conditions (e.g. it will only be necessary to record one set of normalized Stokes parameters for a standard data collection). However, in accordance with the earlier principles, the reference direction $\mathbf{p}$ should be recorded with respect to the crystal lattice, not the laboratory. With the definitions given in $\S 2$, the direction $\mathbf{p}$ can be parametrized by the angle $\xi$ for each reflection record. It should be noted that the status of $\mathbf{p}$ has now changed with respect to the special case of linear polarization discussed in $\$ 2$; it does not necessarily need to correspond to a direction of polarization or to a direction of main linear polarization, but now simply represents the arbitrarily chosen reference direction with respect to which the Stokes parameters $S_{1}$ and $S_{2}$ are defined according to equations (16) and (17). Clearly, in the case of a nearly linearly polarized beam it would be natural (although not compulsory) to chose $\mathbf{p}$ along the direction of polarization, which then corresponds to the simplified treatment presented earlier.

Since the choice of $\mathbf{p}$ is arbitrary, alternative choices will give rise to different normalized Stokes parameters $P_{1}$ and $P_{2}$. Simple geometric considerations show that the set of parameters $\left(P_{1}, P_{2}, P_{3}, \xi\right)$ and $\left(P_{1}^{\prime}, P_{2}^{\prime}, P_{3}^{\prime}, \xi^{\prime}\right)$ are equivalent (characterize the same polarization state) if

$$
\begin{gathered}
P_{1}^{\prime}=P_{1} \cos \left[2\left(\xi^{\prime}-\xi\right)\right]+P_{2} \sin \left[2\left(\xi^{\prime}-\xi\right)\right], \\
P_{2}^{\prime}=-P_{1} \sin \left[2\left(\xi^{\prime}-\xi\right)\right]+P_{2} \cos \left[2\left(\xi^{\prime}-\xi\right)\right], \\
P_{3}^{\prime}=P_{3} .
\end{gathered}
$$

In summary, we propose that the polarization properties of the incident X-ray beam in a diffraction experiment should be specified by the three normalized Stokes parameters $P_{1}, P_{2}$ and $P_{3}$, which are defined with respect to an arbitrary reference direction $\mathbf{p}$ that is fixed in the laboratory frame of reference. The direction $\mathbf{p}$ is then encoded, for each reflection record, with respect to the crystal lattice by using the angle $\xi$.

\subsection{The general form of the polarization correction factor using normalized Stokes parameters}

As an example of the application of Stokes parameters, the general form of the polarization correction factor for a mosaic crystal has been derived by Vaillant (1977). Using the notation and conventions described here, the polarization correction factor for diffracted intensities has the following expression:

$$
\begin{aligned}
\mathcal{P}= & (1 / 2)\left[1+\cos ^{2}(2 \theta)\right]-\left(P_{1} / 2\right) \cos (2 \xi) \sin ^{2}(2 \theta) \\
& -\left(P_{2} / 2\right) \sin (2 \xi) \sin ^{2}(2 \theta) .
\end{aligned}
$$

Clearly, the polarization factor can be computed for a given reflection from the knowledge of the two normalized Stokes parameters $P_{1}$ and $P_{2}$ and the angles $\theta$ and $\xi$. No further information about the experimental geometry is required. This is the most general form of the polarization correction, from which all other expressions reported in the literature (Whittaker, 1953; Ramaseshan \& Ramachandran, 1953; Azároff, 1955; Levy \& Ellison, 1960; Phillips et al., 1977; Kahn et al., 1982), which are often limited to particular geometric settings, can be derived.

If a data set with a sufficient redundancy is available, the Stokes parameters $P_{1}$ and $P_{2}$ could even be refined (along with other scale factors) as part of the data-reduction procedure.

\section{Implementation}

The ideas presented here have been implemented in the software package SHARP (de La Fortelle \& Bricogne, 1997; Bricogne et al., 2003), which is now capable of refining and exploiting AAS properties of anomalously scattering atoms in proteins (Schiltz \& Bricogne, 2008). The auxiliary program SCALA2SHARP (Schiltz \& Bricogne, 2007) prepares a multirecord MTZ data file produced by the CCP4 (Collaborative Computational Project, Number 4, 1994) program SCALA (Evans, 1993) for input to SHARP. In particular, SCALA2$S H A R P$ adds data columns containing $\theta, \psi$ and $\xi$ for each measurement to the reflection file. SHARP is now able to read these values and internally computes the $\mathbf{s}, \mathbf{s}^{\prime}, \mathbf{p}$ and $\mathbf{p}^{\prime}$ vectors that are needed for the refinement of AAS tensors. In the case of partially and/or elliptically polarized X-rays, the normalized Stokes parameters can be declared as refineable parameters at the Batch level within the hierarchical organization of data implemented in SHARP (de La Fortelle \& Bricogne, 1997).

\section{APPENDIX $A$ Computation of $\psi$ and $\xi$}

In general, any data-processing program encodes the crystal orientation in the form of a matrix $[A]=[\mathrm{U}][\mathrm{B}]$ (Busing \& 
Levy, 1967). [B] is a pure orthogonalization matrix, which transforms the coordinates of a vector $\mathbf{v}$ in the reciprocal lattice basis, written as a column matrix $[\mathbf{v}]_{\mathrm{RL}}$, to coordinates in a crystal Cartesian coordinate system, written as a column matrix $[\mathbf{v}]_{\mathrm{CC}}$ :

$$
[\mathbf{v}]_{\mathrm{CC}}=[\mathrm{B}][\mathbf{v}]_{\mathrm{RL}} .
$$

In particular, for a scattering vector $\mathbf{H}$,

$$
[\mathbf{H}]_{\mathrm{CC}}=[\mathrm{B}]\left(\begin{array}{c}
h \\
k \\
l
\end{array}\right) .
$$

The exact form of [B] depends on the choice of the orthogonalization convention. Most data processing program use the original (Busing \& Levy, 1967) convention, which sets the $X$ axis of the Cartesian coordinate system parallel to $\mathbf{a}^{*}$ and its $Z$ axis parallel to c. In some data-processing programs, the entries of the [B] matrix are, however, multiplied by the wavelength $\lambda$, ostensibly to make them dimensionless.

$[\mathrm{U}]$ is a pure rotation matrix, which transforms the coordinates of a vector $\mathbf{v}$ in the crystal Cartesian basis to coordinates in a laboratory Cartesian coordinate system, written as a column matrix $[\mathbf{v}]_{\mathrm{Lab}}$, at a reference position $\left(^{\circ}\right)$ for the diffractometer angles:

$$
[\mathbf{v}]_{\mathrm{Lab}}^{\circ}=[\mathrm{U}][\mathbf{v}]_{\mathrm{CC}} \text {. }
$$

The exact form of $[U]$ depends on the choice of the laboratoryfixed coordinate system. The conventions used in some dataprocessing programs are summarized in Appendix $B$.

The rotations generated by the goniometer axes are represented by an orthogonal matrix denoted as $[\Phi]$, so that

$$
[\mathbf{v}]_{\mathrm{Lab}}=[\Phi][\mathbf{v}]_{\mathrm{Lab}}^{\circ} \text {. }
$$

The exact form of $[\Phi]$ depends on the choice of the laboratoryfixed coordinate system, on the design of the goniometer instrument, and on the conventions used to define the rotation senses (clockwise or anticlockwise) and zero positions of the goniometer axes. For a certain set of diffractometer angles $\boldsymbol{\phi}=(\omega, \kappa, \chi, \varphi, \ldots)$ we then have

$$
[\mathbf{v}]_{\mathrm{Lab}}=[\Phi(\boldsymbol{\phi})][\mathrm{U}][\mathrm{B}][\mathbf{v}]_{\mathrm{RL}}
$$

and, because of equation (27),

$$
[\mathbf{v}]_{\mathrm{Lab}}=[\Phi(\boldsymbol{\phi})][\mathrm{U}][\mathbf{v}]_{\mathrm{CC}}
$$

The coordinates of the incident beam direction $\mathbf{s}$ and beam polarization direction $\mathbf{p}$ in the laboratory coordinate system must be known (they are usually constant). They can then be converted to crystal Cartesian coordinates for each reflection measurement, knowing the diffractometer angles $\boldsymbol{\phi}$ :

$$
\begin{aligned}
& {[\mathbf{s}]_{\mathrm{CC}}={ }^{\mathrm{t}}[\mathrm{U}]^{\mathrm{t}}[\Phi(\boldsymbol{\phi})][\mathbf{s}]_{\mathrm{Lab}},} \\
& {[\mathbf{p}]_{\mathrm{CC}}={ }^{\mathrm{t}}[\mathrm{U}]{ }^{\mathrm{t}}[\Phi(\boldsymbol{\phi})][\mathbf{p}]_{\mathrm{Lab}},}
\end{aligned}
$$

where the left superscript $\mathrm{t}$ denotes matrix transposition.

Once the $[\mathbf{H}]_{\mathrm{CC}},[\mathbf{s}]_{\mathrm{CC}}$ and $[\mathbf{p}]_{\mathrm{CC}}$ matrices have been computed, all the vectors required for the subsequent calculations are available as column matrices of components in the same crystal Cartesian coordinate system. The angles $\theta, \psi$ and $\xi$ can then be computed by the following sequence of calculations:

(i) First, the direction of the diffracted beam is computed by application of the diffraction condition, knowing the wavelength $\lambda$ :

$$
\mathbf{s}^{\prime}=\mathbf{s}+\lambda \mathbf{H}
$$

(ii) The right-handed basis $(\mathbf{e}, \mathbf{f}, \mathbf{g})$ can then be constructed:

$$
\begin{gathered}
\mathbf{e}=-\left(\mathbf{s}^{\prime}+\mathbf{s}\right) /\left|\mathbf{s}^{\prime}+\mathbf{s}\right|, \\
\mathbf{f}=\mathbf{H} /|\mathbf{H}|, \\
\mathbf{g}=\mathbf{e} \times \mathbf{f} .
\end{gathered}
$$

(iii) The reference direction $\mathbf{q}=\mathbf{Q} /|\mathbf{Q}|$ is computed according to the Schwarzenbach \& Flack (1989) convention [equation (2)]. Note that the components of $\mathbf{Q}$ given by equation (2) are expressed in the direct-lattice basis. The conversion to crystal Cartesian coordinates is given by

$$
[\mathbf{Q}]_{\mathrm{CC}}=\left({ }^{\mathrm{t}}[\mathrm{B}]\right)^{-1}\left(\begin{array}{l}
h \\
h \\
0
\end{array}\right)
$$

if $h=k=l$ or

$$
[\mathbf{Q}]_{\mathrm{CC}}=\left({ }^{\mathrm{t}}[\mathrm{B}]\right)^{-1}\left(\begin{array}{c}
k-l \\
l-h \\
h-k
\end{array}\right)
$$

otherwise.

(iv) The angles $\theta$ and $\psi$ can then be computed, respectively, by

$$
\begin{gathered}
\cos \theta=-\mathbf{s} \cdot \mathbf{e}, \\
\sin \theta=\mathbf{s} \cdot \mathbf{f},
\end{gathered}
$$

and

$$
\begin{aligned}
& \cos \psi=\mathbf{q} \cdot \mathbf{g}, \\
& \sin \psi=\mathbf{q} \cdot \mathbf{e} .
\end{aligned}
$$

(v) Finally, the reference vector $\mathbf{t}$ and the $\xi$ angle are computed by

$$
\mathbf{t}=-\sin \theta \mathbf{e}+\cos \theta \mathbf{f}
$$

and

$$
\begin{aligned}
& \cos \xi=\mathbf{p} \cdot \mathbf{t}, \\
& \sin \xi=\mathbf{p} \cdot \mathbf{g} .
\end{aligned}
$$

\section{APPENDIX $B$ Laboratory coordinate systems used by some area- detector data-processing programs}

(i) The program MOSFLM (Leslie, 1993) uses a laboratory coordinate system $\mathbf{e}_{x}, \mathbf{e}_{y}, \mathbf{e}_{z}$ that sets $\mathbf{e}_{x}$ parallel to the incident beam direction $\mathbf{s}$ and pointing in the same direction; $\mathbf{e}_{z}$ is set 
parallel to the rotation axis (which is assumed to be perpendicular to s) and its direction is chosen so that a positive rotation is clockwise when viewed from the origin towards the tip of $\mathbf{e}_{z} ; \mathbf{e}_{y}$ is chosen so as to complete a right-handed orthogonal system. Thus, the coordinates of $\mathbf{s}$ in this laboratory system are always

$$
[\mathbf{s}]_{\mathrm{Lab}}=\left(\begin{array}{c}
1 \\
0 \\
0
\end{array}\right),
$$

but the coordinates of $\mathbf{p}$ depend on its orientation with respect to the rotation axis. If we designate $\eta$ the angle between $\mathbf{p}$ and $\mathbf{e}_{z}$, we can write

$$
[\mathbf{p}]_{\mathrm{Lab}}=\left(\begin{array}{c}
0 \\
\sin \eta \\
\cos \eta
\end{array}\right) .
$$

Thus, on a synchrotron beamline (where $\mathbf{p}$ is in the horizontal plane), a horizontal rotation axis will give $\eta=0$, and for a vertical rotation axis we have $\eta=90^{\circ}$.

(ii) The program $D E N Z O$, which is part of the $H K L$ package (Otwinowski \& Minor, 1997), uses a laboratory coordinate system $\mathbf{e}_{x}, \mathbf{e}_{y}, \mathbf{e}_{z}$ that sets $\mathbf{e}_{z}$ parallel to the incident beam direction $\mathbf{s}$ and $\mathbf{e}_{x}$ parallel to the rotation axis; $\mathbf{e}_{y}$ is chosen so as to complete a right-handed orthogonal system.

(iii) The program $X D S$ (Kabsch, 1988) allows the user free choice of a right-handed orthonormal laboratory coordinate system. The incident beam direction and the direction of the rotation axis are then specified by their components with respect to the chosen coordinate system. The direction of $\mathrm{X}$-ray polarization is not specified directly, but rather the components of the normal to a plane containing the polarization direction (this corresponds to $\mathbf{p}_{\perp}$ in our notation).

(iv) The convention defined for the crystallographic binary file and image-supporting crystallographic information file (CBF/imgCIF) representations is as follows (Bernstein, 2005). The data items in the AXIS category record the information required to describe the goniometer, detector, source and other axes needed to specify a data-collection setup. These vectors are referred to a right-handed laboratory coordinate system with its origin at the specimen. The $X$ axis of this system is aligned to the mechanical axis pointing from the specimen along the principal axis of the goniometer. The $Z$ axis is the component of the source axis (defined to be the axis running from the sample to the source) orthogonal to the $X$ axis. The $Y$ axis completes an orthogonal right-handed system.

\section{APPENDIX $C$}

\section{Computation of $s, s^{\prime}$ and $p$ from $h, k, I, \theta, \psi$ and $\xi$}

We here summarize the formulae that allow one to compute, from the six values $h, k, l, \theta, \psi$ and $\xi$ recorded for each reflection measurement, the diffraction geometry.

(i) First, the scattering vector $\mathbf{H}$ is computed from the $(h k l)$ indices. Similarly, the reference direction $\mathbf{q}=\mathbf{Q} /|\mathbf{Q}|$ is computed by using the Schwarzenbach \& Flack (1989) convention [equation (2)]. These computations only require knowledge of the unit-cell parameters.

(ii) Knowledge of the $\psi$ angle allows one to construct the right-handed basis (e, $\mathbf{f}, \mathbf{g})$ :

$$
\begin{gathered}
\mathbf{f}=\mathbf{H} /|\mathbf{H}|, \\
\mathbf{e}=\sin \psi \mathbf{q}+\cos \psi(\mathbf{f} \times \mathbf{q}), \\
\mathbf{g}=\mathbf{e} \times \mathbf{f} .
\end{gathered}
$$

(iii) Knowledge of the $\theta$ angle allows one to construct the reference vector $\mathbf{t}$ by applying equation (7).

(iv) Finally, the vectors $\mathbf{s}, \mathbf{s}^{\prime}$ and $\mathbf{p}$, which represent directions of physical quantities, can be computed as

$$
\begin{gathered}
\mathbf{s}=-\cos \theta \mathbf{e}-\sin \theta \mathbf{f}, \\
\mathbf{s}^{\prime}=-\cos \theta \mathbf{e}+\sin \theta \mathbf{f}, \\
\mathbf{p}=\cos \xi \mathbf{t}+\sin \xi \mathbf{g} .
\end{gathered}
$$

(v) If needed, the vectors $\mathbf{p}^{\prime}, \mathbf{p}_{\perp}$ and $\mathbf{p}_{\perp}^{\prime}$ can then be computed from equations (8), (10) and (11), respectively.

All these vectors can be computed as column matrices of components in a crystal-based coordinate system. The choice of which system the end-user or software decides to employ is completely free, since the definition of the $\theta, \psi$ and $\xi$ angles is not attached to any particular coordinate system.

We thank Dieter Schwarzenbach for careful reading of the manuscript.

\section{References}

Azároff, L. V. (1955). Acta Cryst. 8, 701-704.

Bathow, G., Freytag, E. \& Haensel, R. (1966). J. Appl. Phys. 37, 3449 3454.

Bernstein, H. J. (2005). International Tables for Crystallography, Vol. G, ch. 3.7, edited by S. Hall \& B. McMahon, pp. 199-208. Dordrecht: Springer.

Blessing, R. H. (1995). Acta Cryst. A51, 33-38.

Blume, M. \& Gibbs, D. (1988). Phys Rev. B, 37, 1779-1789.

Born, M. \& Wolf, E. (1959). Principles of Optics. London: Pergamon Press.

Bricogne, G., Vonrhein, C., Flensburg, C., Schiltz, M. \& Paciorek, W. (2003). Acta Cryst. D59, 2023-2030.

Brunel, M., Patrat, G., de Bergevin, F., Rousseaux, F. \& Lemonnier, M. (1983). Acta Cryst. A39, 84-88.

Busing, W. R. \& Levy, H. A. (1967). Acta Cryst. 22, 457-464.

Collaborative Computational Project, Number 4 (1994). Acta Cryst. D50, 760-763.

Dmitrienko, V. E., Ishida, K., Kirfel, A. \& Ovchinnikova, E. N. (2005). Acta Cryst. A61, 481-493.

Evans, P. R. (1993). Proceedings of CCP4 Study Weekend on Data Collection and Processing, pp. 114-122. Daresbury: SERC Daresbury Laboratory.

Fanchon, E. \& Hendrickson, W. A. (1990). Acta Cryst. A46, 809-820.

Giles, C., Malgrange, C., Goulon, J., de Bergevin, F., Vettier, C., Dartyge, E., Fontaine, A., Giorgetti, C. \& Pizzini, S. (1994). J. Appl. Cryst. 27, 232-240. 
Hirano, K., Ishikawa, T. \& Kikuta, S. (1995). Rev. Sci. Instrum. 66, 1604-1609.

Ishikawa, T., Hirano, K. \& Kikuta, S. (1991). J. Appl. Cryst. 24, $982-$ 986.

Kabsch, W. (1988). J. Appl. Cryst. 21, 916-924.

Kahn, R., Fourme, R., Gadet, A., Janin, J., Dumas, C. \& André, D. (1982). J. Appl. Cryst. 15, 330-337.

Kirfel, A., Petcov, A. \& Eichhorn, K. (1991). Acta Cryst. A47, 180195.

La Fortelle, E. de \& Bricogne, G. (1997). Methods in Enzymology, Vol. 276, Macromolecular Crystallography, edited by C. W. Carter Jr \& R. M. Sweet, pp. 472-494. New York: Academic Press.

Leslie, A. G. W. (1993). Proceedings of CCP4 Study Weekend on Data Collection and Processing, pp. 44-51. Daresbury: SERC Daresbury Laboratory.

Levy, H. A. \& Ellison, R. D. (1960). Acta Cryst. 13, 270-271.

Lovesey, S. W. \& Collins, S. P. (1996). X-ray Scattering and Absorption by Magnetic Materials. Oxford University Press.

Lovesey, S. W. \& Collins, S. P. (2001). J. Synchrotron Rad. 8, 10651077.

Materlik, G. \& Suortti, P. (1984). J. Appl. Cryst. 17, 7-12.
Otwinowski, Z. \& Minor, W. (1997). Methods in Enzymology, Vol. 276, Macromolecular Crystallography, edited by C. W. Carter Jr \& R. M. Sweet, pp. 307-326. New York: Academic Press.

Phillips, J. C., Wlodawer, A., Goodfellow, J. M., Watenpaugh, K. D., Sieker, L. C., Jensen, L. H. \& Hodgson, K. O. (1977). Acta Cryst. A33, 445-455.

Poincaré, H. (1889). Leçons sur la Théorie Mathématique de la Lumière, edited by J. Blondin. Paris: Georges Carré.

Ramaseshan, S. \& Ramachandran, G. N. (1953). Acta Cryst. 6, 364-365. Schiltz, M. \& Bricogne, G. (2007). J. Synchrotron Rad. 14, 34-42.

Schiltz, M. \& Bricogne, G. (2008). Acta Cryst. D64, 711-729.

Schwarzenbach, D. \& Flack, H. D. (1989). J. Appl. Cryst. 22, 601-605. Sheldrick, G. M. (2008). Acta Cryst. A64, 112-122.

Shen, Q. \& Finkelstein, D. (1992). Phys. Rev. B, 45, 5075-5078.

Stokes, G. G. (1852). Trans. Camb. Philos. Soc. 9, 399-416.

Templeton, D. H. \& Templeton, L. K. (1982). Acta Cryst. A38, 62-67.

Templeton, D. H. \& Templeton, L. K. (1988). J. Appl. Cryst. 21, 151-153.

Vaillant, F. (1977). Acta Cryst. A33, 967-970.

Hümmer, K. \& Weckert, E. (1995). Acta Cryst. A51, 431-438.

Weckert, E. \& Hümmer, K. (1997). Acta Cryst. A53, 108-143.

Whittaker, E. J. W. (1953). Acta Cryst. 6, 222-223. 